\title{
Sizing the width and depth of real cracks using laser spot lock-in thermography
}

\author{
by M. Colom*, J. Rodríguez-Aseguinolaza*, A. Mendioroz* and A. Salazar* \\ * Departamento de Física Aplicada I, Escuela de Ingeniería de Bilbao, Universidad del País Vasco UPV/EHU, \\ Plaza Ingeniero Torres Quevedo 1, 48013 Bilbao, Spain, agustin.salazar@ehu.es
}

\begin{abstract}
We have calculated the surface temperature of a sample containing a surface breaking crack, when a modulated laser spot is focused close to it. This temperature has been obtained numerically using discontinuous finite elements, which are well adapted to deal with narrow cracks. We have used this model to analyse a metallic sample containing a fatigue crack, which remains invisible by optics means. In this way, we have obtained simultaneously the depth and width of the crack.
\end{abstract}

\section{Introduction}

Detecting surface breaking cracks by means of infrared thermography requires exciting a lateral heat flux, which is perturbed by the presence of the crack, since it acts as a thermal barrier. That is reason why laser spot thermography was proposed many decades ago as a non-destructive tool for cracks detection [1]. More challenging is to characterize its length, depth $(d)$ and width $(w)$ from the surface temperature recorded by an infrared camera. The length of a crack can be obtained by a post-processing technique that consists in computing the first (or second) spatial derivatives along two perpendicular directions, which reflect the amplitude change rate in the thermogram and thus extracts the crack extension [2]. The other two parameters ( $d$ and $w$ ) are not directly accessible and they must be obtained from a fitting of the surface temperature data to a model.

In this work, first we have obtained the surface temperature of a sample containing a crack when it is excited by a modulated laser spot focused close to the crack. To do this, we have solved the heat diffusion equation numerically. We have developed a code based on discontinuous Galerkin finite elements, which, unlike continuous finite elements, allows dealing with very narrow cracks [3]. Then we have used that code to fit the surface temperature data recorded by an IR camera on a metallic sample containing a fatigue crack. From this fitting we have obtained simultaneously $d$ and $w$.

\section{Theory}

Figure 1a shows the cross section of a semi-infinite and opaque sample containing a very long ( $x$-axis) surface breaking vertical crack of depth $d$ and width $w$. It is illuminated by a laser beam of Gaussian profile focused to a radius $a$, whose centre is at the $y$-axis, at a distance $l$ from the crack. The laser beam is modulated at a frequency $f$. We have calculated the surface temperature numerically by solving the heat diffusion equation using discontinuous finite elements. The details of the procedure can be found in Reference [4].

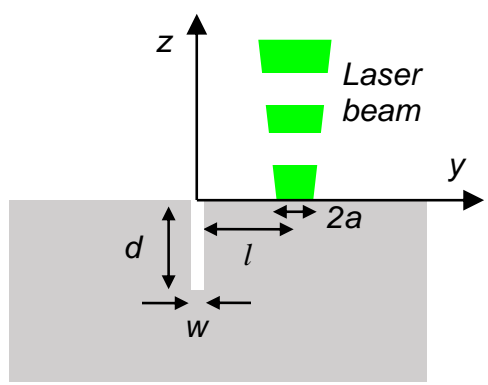

(a)
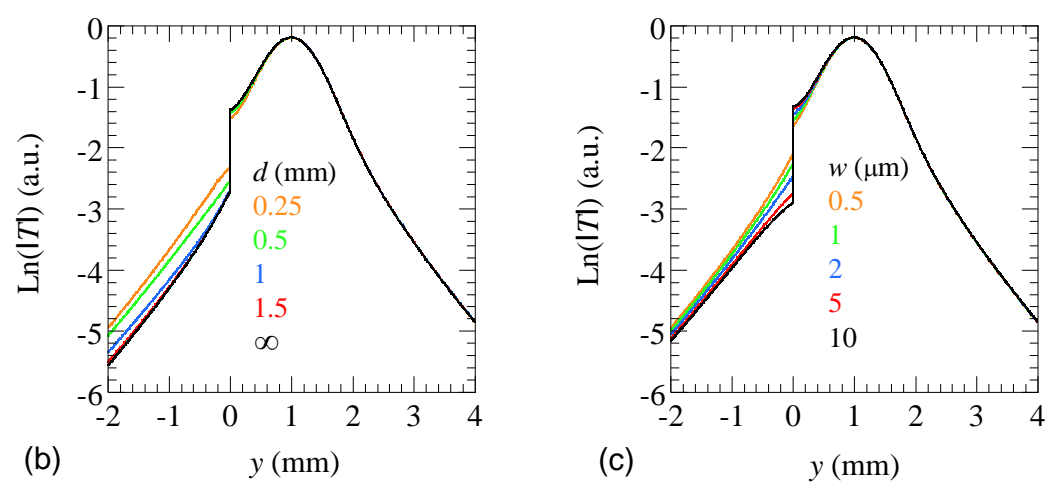

Fig. 1. (a) Cross-section of the cracked sample. (b) Calculated temperature amplitude profile corresponding to an AISI304 sample containing a long crack with $l=1 \mathrm{~mm}, a=0.75 \mathrm{~mm}, f=1 \mathrm{~Hz}$. The width is fixed $w=2.5 \mu \mathrm{m}$ and we change the depth. (c) The same sample and experimental parameters for a fixed crack depth $(d=0.5 \mathrm{~mm})$, changing the crack width. 
In figure $1 \mathrm{~b}$ we plot the numerical simulations of the temperature amplitude profile along the $y$-axis for an AISI304 stainless steel sample $\left(D=4 \mathrm{~mm}^{2} / \mathrm{s}, K=15 \mathrm{Wm}^{-1} \mathrm{~K}^{-1}\right)$ containing a fissure of width $w=2.5 \mu \mathrm{m}$. A logarithmic scale is used to enhance the differences at low temperature values. Calculations are performed for $f=1 \mathrm{~Hz}, a=0.75 \mathrm{~mm}$ and $l=$ $1 \mathrm{~mm}$. Several crack depths are considered. As can be observed, an abrupt temperature jump is observed at the crack position $(y=0)$. Its height increases with the crack depth until saturation is reached: there is a limit depth $d_{\text {lim }}$ which is undistinguishable of an infinite crack. This depth verifies $d_{\text {lim }} \approx \mu=\sqrt{D /(\pi f)}$, the thermal diffusion length. Anyway, it is worth noting that the temperature jump at the crack position does not determine univocally the crack depth, since, as can be observed in figure $1 \mathrm{~b}, d=1 \mathrm{~mm}$ and $d=2 \mathrm{~mm}$ produce the same temperature jump, but the left tail is different for both depths. Figure $1 \mathrm{c}$ is the same as figure $1 \mathrm{~b}$, but fixing the crack depth $(d=0.5 \mathrm{~mm})$ and varying the crack width. The main conclusion of this theoretical analysis is that to retrieve simultaneously depth and width, a fitting of the temperature profile along the $y$-axis must be performed or, what is even better, a fitting of the complete surface thermogram.

\section{Experimental results and discussion}

Figure 2a shows the optical image of a Aluminum 7475-T7351 sample ( $\left.D=60 \mathrm{~mm}^{2} / \mathrm{s}\right)$ containing a drilling hole, that has been subjected to a fatigue test. Fatigue cracks are expected to develop starting from the hole. However, the optical inspection does reveal any fissure. The sample was studied using a conventional laser spot lock-in thermography setup with $f=14 \mathrm{~Hz}$ and $\mathrm{a}=180 \mu \mathrm{m}$. Figure $2 \mathrm{~b}$ shows the summed first-derivative image of the sample along the horizontal and vertical directions, clearly indicating the presence of a crack about $10 \mathrm{~mm}$ long.

In a second step, we fitted the temperature amplitude profiles to the numerical model to retrieve both, width and depth of the crack. We found that the depth is higher than $1.5 \mathrm{~mm}$, the $d_{\text {lim }}$ corresponding to the thermal diffusion length at that frequency. Regarding the width, it decreases monotonously from $2 \mu \mathrm{m}$ close to the hole, down to submicronic depths from $3 \mathrm{~mm}$ to $10 \mathrm{~mm}$. It is worth mentioning the outstanding sensitivity of the method, which is able to detect and characterize submicronic fissures. Complementary studies at lower frequencies will allow to better precise the real depth of the crack.

The future trend is to implement this method to be used with a laser spot moving at constant speed, the so-called Flying spot thermography. In this way, the whole sample surface could be scanned in a short time.

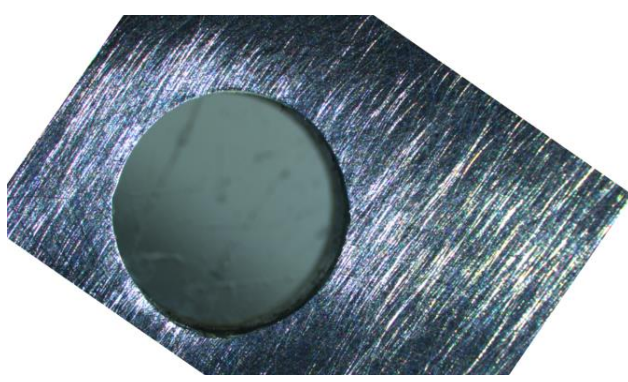

(a)

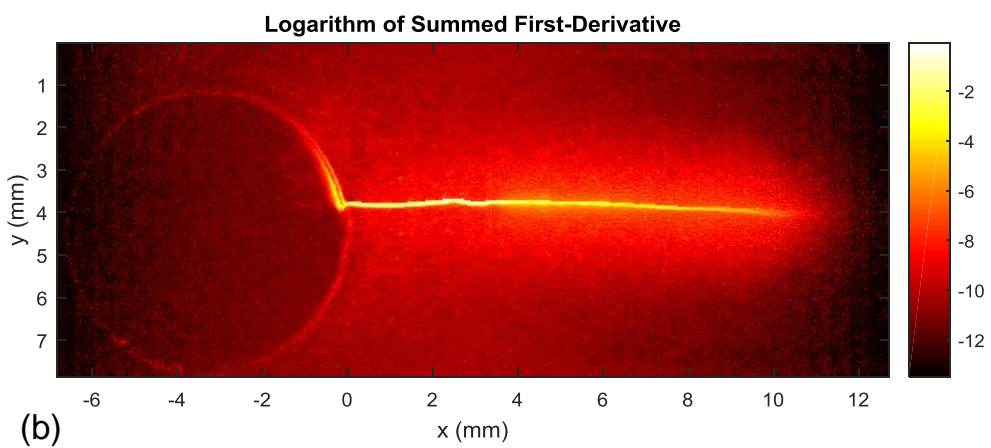

(b)

Fig. 2. (a) Optical image of the sample. No crack is visible. (b) Post-processed thermographic image of the sample using the first-derivative. A $10 \mathrm{~mm}$ long crack is clearly seen.

This work has been supported by Ministerio de Economía y Competitividad, (DPI2016-77719-R, AEI/FEDER, UE), by Universidad del País Vasco UPV/EHU (GIU16/33) and by Gobierno Vasco (PIBA 2018-15).

\section{REFERENCES}

[1] Kubiak E.J., "Infrared detection of fatigue cracks and other near-surface defects". Applied Optics 7, 1743-1747 (1968).

[2] Li T., Almond D.P., Rees D.A.S., "Crack imaging by scanning laser-line thermography and laser-spot thermography". Measurement Science \& Technology 22, 035701 (2011).

[3] Douglas N.A., Brezzi F., Cockburn B., Marini L.D.,"Unified analysis of discontinuous Galerkin methods for elliptic problems". SIAM Journal on Numerical Analysis 39, 1749-1779 (2002).

[4] Celorrio R., Omella A.J., Pech-May N.W., Oleaga A., Mendioroz A., Salazar A., "Vertical cracks characterization using lock-in thermography: II. Finite cracks". Measurement Science \& Technology 25, 115602 (2014). 